\title{
On the Solvability of the Mutual Localization Problem with Anonymous Position Measures
}

\author{
Antonio Franchi, Giuseppe Oriolo, and Paolo Stegagno
}

\begin{abstract}
This paper formulates and investigates a novel problem called Mutual Localization with Anonymous Position Measures. This is an extension of Mutual Localization with Position Measures, with the additional assumption that the identities of the measured robots are not known. A necessary and sufficient condition for the uniqueness of the solution is presented, which requires $O\left(n^{2} / \log n\right)$ to be verified and is based on the notion of rotational symmetry in $\mathbb{R}^{2}$. We also derive the relationship between the number of robots and the number of possible solutions, and classify the solutions in a number of equivalence classes which is linear in $n$. A control law is finally proposed that effectively breaks symmetric formations so as to guarantee unique solvability of the problem is also proposed; its performance is illustrated through simulations.
\end{abstract}

\section{INTRODUCTION}

The Mutual Localization problem (i.e., determining the relative locations in a group of sensing agents) has received a lot of attention in recent years [1], [2], [3], due to the fact that its solution is essential to perform many multirobot tasks, e.g., coverage and deployment [4], exploration and map building [5], formation control [6], surveillance and monitoring [7], escorting and entrapment [8]. Great relevance has been given in the literature to the determination of conditions for the uniqueness of the solution given the type of available measures, represented with a measuring graph. In [1] the range measurement case is addressed and the uniqueness of the solution is connected to various concepts of rigidity, while in [2] this analysis is extended to the position measurement case. In [3], the determination of the sensor orientation is included in the problem formulation, and it is proven that the uniqueness of the solution is guaranteed if the measuring graph is complete. While all these papers address a static (i.e., instantaneous) version of the mutual localization problem, a dynamic version is studied in [9] via the analysis of observability properties.

In this paper, we consider an extension of the above static problem, called Mutual Localization with Anonymous Position Measures, by adding the assumption that the set of relative position measurements of each robot is not labeled, in the sense that each measurement comes without the identity of the measured robot. This situation typically arises when the robot detection system is based on a feature extraction module that looks for physical characteristics that are common to all robots, e.g., size, color, or shape in a group of identical robots. For example, in [10] we have made use of

The Authors are with the Dipartimento di Informatica e Sistemistica Università di Roma La Sapienza, Roma, Via Ariosto 25, 00185 Italy. Email: \{franchi, oriolo, stegagno\}@dis.uniroma1.it a robot detector based on a laser range finder, that is unable to provide the identity of the measured robot.

In practical applications, adverse environmental conditions (unstable light or darkness, rain, fog, etc.) may hide the distinguishing features which are commonly used for identification. Hence, the possibility of relying on a localization system which does not require the identities of the robot makes the system more robust. On the other hand, resemblance of the group members can be essential in missions where the leader identity must remain secret for security or disguising purposes, e.g., in escorting or intrusion.

In opposition to the standard Mutual Localization with Position Measures, the problem of Mutual Localization with Anonymous Position Measures may have more than one solution even if the measuring graph is complete. For example, consider the case of $n$ robots that are arranged over the vertexes of a regular $n$-gon, aiming their 'noses' cyclically at each other. Since the measures of all robots are identical, all the $n$ ! vertex associations corresponding to the permutations of the sequence $\{1, \ldots, n\}$ are feasible. Even considering solutions obtained by roto-translations to be equivalent, as they clearly are in the absence of absolute localization, there are still $(n-1)$ ! feasible labeled formations.

The importance of this problem goes beyond the deterministic case. In fact, if the localization problem is formulated in a stochastic setting to take into account measurement noise, the cases with multiple solutions correspond to large uncertainties in the probability density of the solution. This happens already when the group is close to a configuration that gives rise to multiple solutions in the deterministic case. It is also worth noting that multiple solutions appear, in particular, when the group is arranged along a regular pattern; this situation is not rare in multi-robot systems, since many collective motion controllers attempt to achieve exactly this kind of formation, see e.g. [6].

A loosely related problem is the registration of multiple representations of the same scene [11] and the unknown data association of landmarks in SLAM [12].

The paper is organized as follows. The problem under consideration is formalized in Sect. II. In Sect. III we characterize the situations in which the problem has multiple solutions, establish the relationship between the number of possible solutions and the number of robots, and propose an efficient representation of the solutions. A control law that breaks symmetric formations so as to guarantee unique solvability of the problem is proposed in Sect. IV, and is validated through simulations in Sect. V. Finally, some ongoing work is discussed in Sect. VII. 


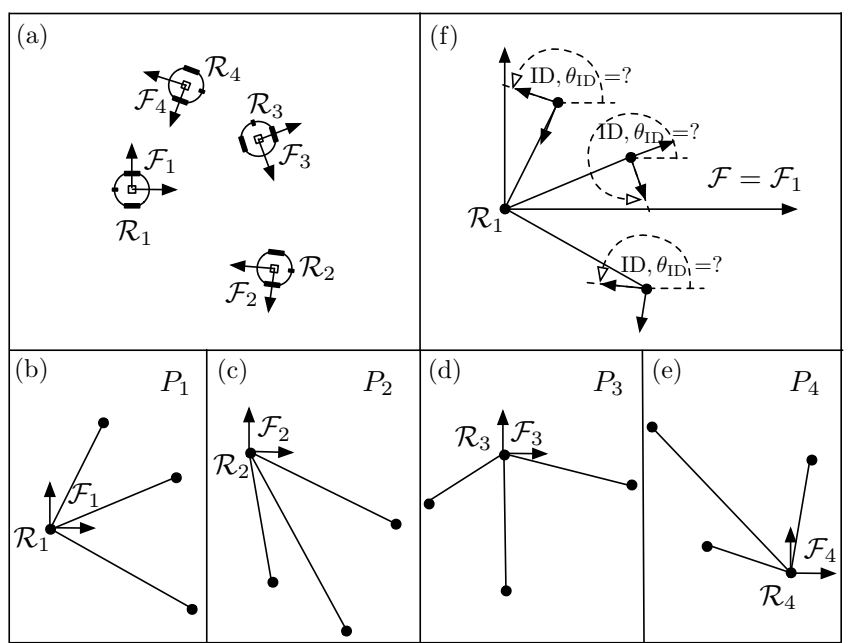

Fig. 1. Mutual Localization with Anonymous Position Measures. (a) Each robot expresses its measures in an attached frame $\mathcal{F}_{i}$ (b-e) $P_{1}, \ldots, P_{4}$ are the observations of the robots $\mathcal{R}_{1}, \ldots, \mathcal{R}_{4}$, respectively (f) with the reference frame $\mathcal{F}$ chosen w.l.o.g. to be $\mathcal{F}_{1}$, the problem is to reconstruct the formation (including orientations and identities) of the robots.

\section{Problem Formulation}

We have a group of $n$ single-body robots in a certain spatial arrangement on the plane. Each robot is equipped with a sensor that provides the positions (not the orientations) of the other robots with respect to itself; these positions are anonymous, i.e., they are not labeled with the identity of the robots. We want to identify conditions under which the spatial arrangement of the group can be uniquely reconstructed (up to roto-translations) from the knowledge of all sensory data. Below, we give a formal statement of this problem.

The $i$-th robot $\mathcal{R}_{i}, i=1, \ldots, n$, is a planar rigid body with an attached frame $\mathcal{F}_{i}$ (see Fig. 1a). The pose $x_{i}=\left(p_{i}, \theta_{i}\right)$ of $\mathcal{R}_{i}$ is an element of $\mathbb{R}^{2} \times S^{1}$, with $p_{i}$ representing the origin ${ }^{1}$ of $\mathcal{F}_{i}$ expressed in a reference frame $\mathcal{F}$ and $\theta_{i}$ the orientation of $\mathcal{F}_{i}$ w.r.t. $\mathcal{F}$. Since $\mathbb{R}^{2} \times S^{1}$ is homeomorphic to $S E(2)$, any pose may also be interpreted as a roto-translation.

A formation is a set of $n$ poses $\left\{x_{1}, \ldots, x_{n}\right\}$ in $\mathcal{F}$, with $x_{i}$ assigned to $\mathcal{R}_{i}$. Since we are interested in computing the group formation up to roto-translations, we can set w.l.o.g. $\mathcal{F}=\mathcal{F}_{1}$, so that $x_{1}=\left(\left(\begin{array}{ll}0 & 0\end{array}\right)^{T}, 0\right)$. This means that all formations will be expressed in the frame attached to $\mathcal{R}_{1}$. Clearly, all results can be expressed in another frame $\mathcal{F}^{\prime}$ provided that the pose of $\mathcal{R}_{1}$ w.r.t. $\mathcal{F}^{\prime}$ is known.

Let $R(\phi) \in S O(2)$ denote the rotation matrix associated to an angle $\phi$. As in [11] and [13], we denote by $x_{a} \oplus x_{b}$ and $x_{a} \ominus x_{b}$, respectively, the composition and the inverse composition of two poses, defined by the following formulas:

$$
\begin{aligned}
& x_{a} \oplus x_{b}=\left(p_{a}+R\left(\theta_{a}\right) p_{b}, \theta_{a}+\theta_{b}\right) \\
& x_{a} \ominus x_{b}=\left(R\left(-\theta_{b}\right)\left(p_{a}-p_{b}\right), \theta_{a}-\theta_{b}\right) .
\end{aligned}
$$

Operators $\oplus$ and $\ominus$ are also used to compose twodimensional position vectors with three-dimensional poses.

\footnotetext{
${ }^{1}$ For simplicity, we use the same symbol (e.g., $p$ ) to indicate a point and its Cartesian coordinates; the actual meaning will be clear from the context.
}

In particular, given the coordinates $p$ of a point expressed in $\mathcal{F}_{i}$, whose pose w.r.t. $\mathcal{F}$ is $x_{i}$, the operation $x_{i} \oplus p$ gives the coordinates of the same point expressed in $\mathcal{F}$. Conversely, given $x_{i}$ and the coordinates $p$ of a point expressed in $\mathcal{F}$, the operation $p \ominus x_{i}$ gives the coordinates of the same point expressed in $\mathcal{F}_{i}$, whose pose w.r.t. $\mathcal{F}$ is $x_{i}$. These operators may also be used with a set $P$ of points, by letting $x_{i} \oplus P:=\left\{x_{i} \oplus p \mid p \in P\right\}$, and $P \ominus x_{i}:=\left\{p \ominus x_{i} \mid p \in P\right\}$.

An observation $P_{i}$ is a set of $n$ distinct points in $\mathbb{R}^{2}$, one of which is always the origin. It represents the positions of the robots as measured by the $i$-th robot, i.e., relative to $\mathcal{F}_{i}$. Apart from the origin, which stands for $\mathcal{R}_{i}$ itself, $P_{i}$ does not convey any information about the identity of the robot located at a certain point (anonymity), nor about its orientation. Note also that all the observations of a given group are the same up to roto-translations. See Fig. 1b-e for examples of observations.

Problem 1 (Mutual Localization with Anonymous Position Measures). Given $n$ observations $P_{1}, \ldots, P_{n}$, find all the possible pairs of functions

$$
\begin{aligned}
& \hat{p}:\{2, \ldots, n\} \rightarrow P_{1} \backslash(00)^{T} \\
& \hat{\theta}:\{2, \ldots, n\} \rightarrow[0,2 \pi),
\end{aligned}
$$

with $\hat{p}$ bijective, such that

$$
P_{1} \ominus \hat{x}_{i}=P_{i} \quad i=2, \ldots, n,
$$

where $\hat{x}_{i}:=(\hat{p}(i), \hat{\theta}(i))$.

Function $\hat{p}$ assigns each point of $P_{1}$ (with the exception of the origin) to one and only one robot in $\left\{\mathcal{R}_{2}, \ldots, \mathcal{R}_{n}\right\}$, whose orientation is then defined by $\hat{\theta}$ (see Fig. 1b). Note that $\mathcal{R}_{1}$ is directly associated to the origin, with orientation equal to zero, in all solutions to the problem. Stated differently, Problem 1 consists in finding all the formations $\left\{\hat{x}_{1}=\right.$ $\left.\left(\left(\begin{array}{ll}0 & 0\end{array}\right)^{T}, 0\right), \hat{x}_{2} \ldots, \hat{x}_{n}\right\}$ that are compatible with the given observations, i.e., satisfy (1) (see Fig. 1f).

In general, a solution to Problem 1 may exist or not. In the following, we assume that each observation $P_{i}, i=1, \ldots, n$, has been gathered by robot $\mathcal{R}_{i}$ with reference to the same spatial arrangement of the group. This is sufficient to claim that Problem 1 admits at least one solution.

\section{Unique Solvability, Structure AND Number OF SOLUTIONS}

In this section we give a necessary and sufficient condition for the unique solvability of Problem 1 (Proposition 1), an associated test (Proposition 2), and a quantitative and qualitative characterization of the solutions (Propositions 3 and 4). In particular, we show that the problem is uniquely solvable if and only if the set of points represented by observation $P_{1}$ does not have a rotational symmetry (remember that all observations are the same up to roto-translations). Furthermore, we show that in the case of non-unique solvability the number of solutions increases factorially with $n$, the number of robots. To establish these results, we first recall a few basic concepts on rotational symmetry. 

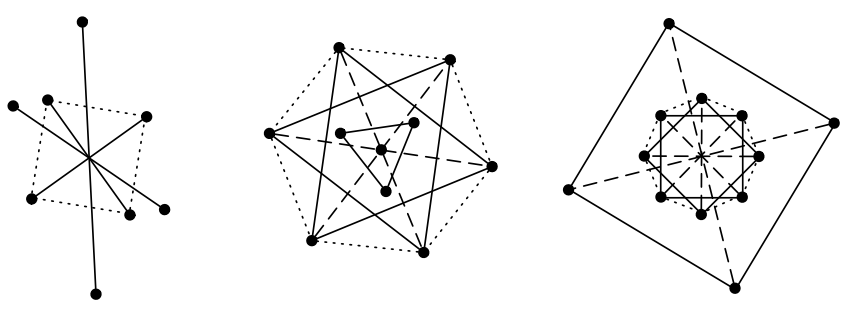

Fig. 2. Three rotational symmetric sets of points. From left to right, the associated proper symmetric groups are respectively $C_{2}, C_{3}$ and $C_{4}$. Note that only the second set contains its centroid. Solid line segments join points that belong to the same set of the rotational symmetric partition. Dotted line segments show the presence of partial higher-degree symmetries which are not relevant for the analysis: from left to right, they identify respectively a square, an hexagon and an octagon. Dashed line segments meet at the centroid of each set.

\section{A. A brush-up on rotational symmetry}

Consider a set of $n$ points $P \subset \mathbb{R}^{2}$. Let $\mathcal{S}_{P}$ denote the proper symmetry group of $P$, i.e., the subgroup of its orientationpreserving isometries (roto-translations) under which it is invariant. It is known from symmetry group theory [14] that, since $P$ is a bounded set, $\mathcal{S}_{P}$ can be represented as a subgroup of $S O(2)$ (the group of planar rotations), by choosing the origin to be its fixed point, i.e., the centroid ${ }^{2}$ of $P$. In particular, there exists a positive integer $l$ such that $\mathcal{S}_{P}=C_{l}$, where $C_{l}$ is the cyclic group of order $l$, whose generator is the rotation of $2 \pi / l$. $P$ is said to be rotational symmetric if $\mathcal{S}_{P} \neq C_{1}$, where $C_{1}$ is the trivial group containing only the identity operation.

Assume that $\mathcal{S}_{P}=C_{l}$ and let $c$ be the centroid of $P$. Denote by $q_{\phi}=(c-R(\phi) c, \phi)$ the rotation by an angle $\phi$ around $c$, and in particular by

$$
q_{k}:=(c-R(2 k \pi / l) c, 2 k \pi / l),
$$

the rotation by $2 k \pi / l$, for $k=0,1, \ldots, l-1$. We have then $P=P \ominus q_{k}=q_{k} \oplus P$, for $k=0,1, \ldots, l-1$. Note that rotational symmetry is invariant under isometries: if $P$ is rotational symmetric, also $P \ominus x$ is rotational symmetric, for any $x \in S E(2)$. Examples of rotational symmetric sets of points are shown in Fig. 2.

The following Lemma establishes a property which is valid for any finite set of points and has an important role in the study of the unique solvability of Problem 1.

Lemma 1 (Rotational Symmetric Partition). For each set $P$ of $n$ points for which $\mathcal{S}_{P}=C_{l}$, there exists a partition $\mathcal{E}_{P}=\left\{E_{1}, \ldots, E_{m}\right\}$ of $P$ such that $E_{j}, j=1, \ldots, m$, is invariant under any rotation in $C_{l}$ around the centroid $c$, i.e.,

$$
E_{j}=E_{j} \ominus q_{k}, \quad k=0,1 \ldots, l-1 .
$$

If $c \notin P$, then $l$ divides $n, m=n / l$, and the cardinality of each subset of the partition $\mathcal{E}_{P}$ is $l$. If $c \in P$, then l divides $n-1, m=1+(n-1) / l$ and the cardinality of each subset in $\mathcal{E}_{P} \backslash\{c\}$ is $l$.

\footnotetext{
${ }^{2}$ Since after any rotation in $\mathcal{S}_{P}$ the set of points $P$ remains the same, also the centroid remains the same, hence the centroid is the fixed point.
}

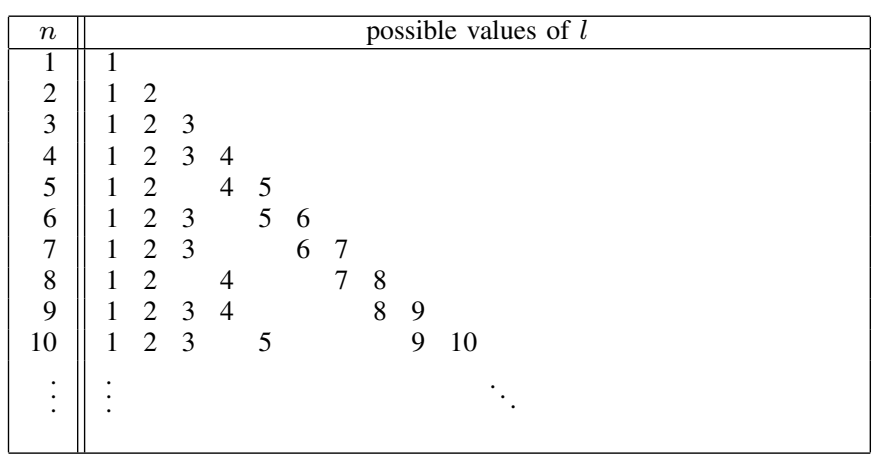

Fig. 3. The possible values of the integer $l$ for the cyclic groups $C_{l}$ that can be the proper symmetry groups of a set $P$ of $n$ points. Note that, since $P$ can always be non-rotational symmetric, $l=1$ is ubiquitous. Also, $l=2$ is always possible since for any odd value of $n$ one point can be always placed in the centroid.

Proof: Suppose w.l.o.g. that $c$ is the origin. Chosen a point $p \in P \backslash\{c\}$, the set $E(p)$ of all points obtained applying an element of $C_{l}$ to $p$ is a subset of $P$ by definition. Clearly, $E(p)$ has cardinality $l$ and is invariant under $C_{l}$. Now choose a point $p^{\prime}$ in $P \backslash E(p)$, repeat the above construction to obtain $E\left(p^{\prime}\right)$, and proceed as before. If $c \notin P$, the collection of all the distinct sets $E(p)$ for all $p \in P$ gives the subsets $E_{1}, \ldots, E_{m}$ of the partition $\mathcal{E}_{P}$, with $m=n / l$. On the other hand, if $c \in P$ then set $E(c)$ is a singleton and must be added to the previous collection, which consists in this case of $(n-1) / l$ subsets.

Figure 2 shows the partitions for three different rotational symmetric set of points, while in Fig. 3 the possible values of $l$ are tabulated for sets of $n=1, \ldots, 10$ points. Limit cases are $l=1$ (the set of points is not rotational symmetric, and the partition consists of $n$ singletons) and $l=n$ (the set of points may be a regular $n$-gon, and the partition consists if a single set containing all the points in $P$ ).

\section{B. Unique solvability of Problem 1}

In the rest of this section, we assume that $\mathcal{S}_{P_{1}}=C_{l}$ and denote by $c$ the centroid of $P_{1}$. The role of rotational symmetry in the unique solvability of Problem 1 is clarified by the following result.

Proposition 1 (Unique Solvability). Assume that Problem 1 admits a solution. The solution is unique if and only if $P_{1}$ is not rotational symmetric.

Proof: Assume that Problem 1 admits multiple solutions. Then there exists $i$ and two poses $\hat{x}_{i}^{\prime}$ and $\hat{x}_{i}^{\prime \prime} \neq \hat{x}_{i}^{\prime}$ such that $P_{1} \ominus \hat{x}_{i}^{\prime}=P_{i}$ and $P_{1} \ominus \hat{x}_{i}^{\prime \prime}=P_{i}$. Then $P_{1}=\hat{x}_{i}^{\prime \prime} \oplus P_{1} \ominus \hat{x}_{i}^{\prime}$, i.e., there exists a non-zero roto-translation which transforms $P_{1}$ in itself; this means that $P_{1}$ is rotational symmetric. On the other hand, assume that $P_{1}$ is rotational symmetric. Since a solution $\left\{x_{1}, \ldots, x_{n}\right\}$ exists, i.e., $P_{1} \ominus \hat{x}_{i}=P_{i}$, $i=1, \ldots, n$, there exists a non-zero roto-translation $x$ which transforms $P_{1}$ in itself, i.e., $P_{1}=P_{1} \ominus x$. This means that $\left\{x \ominus x_{1}, \ldots, x \ominus x_{n}\right\}$ is also a solution.

Proposition 1 implies that the number of solutions to Problem 1 is invariant with respect to changes in the orientations 
of the robots in the formation (in spite of the fact that the observations change).

Unique solvability may be tested with the aid of the following result.

Proposition 2 (Unique Solvability Test). Denote with $P_{1}(\phi)$ the set of points obtained by rotating the observation $P_{1}$ by an angle $\phi$ around its centroid $c$, i.e.:

$$
P_{1}(\phi):=\left\{R(\phi)(p-c)+c \mid p \in P_{1}\right\} .
$$

If $c \notin P$, Problem 1 has a unique solution if and only if

$$
P_{1} \neq P_{1}(2 \pi / m) \quad \forall m \text { prime factor of } n \text {. }
$$

If $c \in P$, $n$ must be replaced by $n-1$ in (4).

Proof: Since $P_{1}$ has $n$ points, its proper-symmetry group $\mathcal{S}_{P_{1}}$ can only be one of the cyclic groups $C_{1}, \ldots, C_{n}$. In addition, since $C_{l}, 2 \leq l \leq n$, also belongs to any $C_{m}$ with $m$ prime factor of $l$, and $l$ can only be a divisor of $n$ (if $c \notin P_{1}$ ) or $n-1$ (if $c \in P_{1}$ ), it is sufficient to check the rotations that are generators of the cyclic groups $C_{m}$, with $m$ prime factor of $n$ or $n-1$.

Assume that $c \notin P_{1}$. Since condition (4) requires $n$ checks for any value of $m$, the overall complexity of the test is $O(n \cdot \pi(n))$, where the prime-counting function $\pi(n)$ can be approximated by $n / \log (n)$. If $c \in P_{1}$, the complexity is $O((n-1) \cdot \pi(n-1))$.

\section{Structure and number of multiple solutions}

We now turn our attention to the case when there are multiple solutions to Problem 1.

Proposition 3 (Structure of the Solutions). Let $i=2, \ldots, n$. If $\hat{x}_{i}$ is a feasible pose for $\mathcal{R}_{i}$, in the sense that $\hat{x}_{i}=\left(\hat{p}_{i}, \hat{\theta}_{i}\right)$ satisfies (1), then all the non-zero poses obtained as $q_{k} \oplus \hat{x}_{i}$, with $k=0,1, \ldots, l-1$ and $q_{k}$ defined by (2), are feasible for $\mathcal{R}_{i}$, and vice versa.

Proof: Being $P_{1} \ominus \hat{x}_{i}=P_{i}$ and $P_{1}=P_{1} \ominus q_{k}$, we have $\left(q_{k} \oplus P_{1}\right) \ominus \hat{x}_{i}=P_{i}$. Developing the pose compositions for an element $p$ of $P_{1}$ we have that

$$
\begin{aligned}
\left(q_{k} \oplus p\right) \ominus \hat{x}_{i} & =\left(c_{k}+R\left(\phi_{k}\right) p\right) \ominus \hat{x}_{i} \\
& =R\left(-\hat{\theta}_{i}\right)\left(c_{k}+R\left(\phi_{k}\right) p-\hat{p}_{i}\right) \\
& =R\left(-\hat{\theta}_{i}\right) R\left(\phi_{k}\right)\left(p-R\left(-\phi_{k}\right)\left(\hat{p}_{i}-c_{k}\right)\right) \\
& =p \ominus\left(\hat{x}_{i} \ominus q_{k}\right) .
\end{aligned}
$$

Hence $\left(q_{k} \oplus P_{1}\right) \ominus \hat{x}_{i}=P_{1} \ominus\left(\hat{x}_{i} \ominus q_{k}\right)$ and $\hat{x}_{i} \ominus q_{k}$ is a feasible solution, for $k=0,1, \ldots, l-1$, which is equivalent to say that $q_{k} \oplus \hat{x}_{i}$ is a feasible solution, for $k=0,1, \ldots, l-1$. Similarly, it is simple to show that for any other feasible pose $x^{\prime} \oplus \hat{x}_{i}, x^{\prime}$ must belong to $\left\{q_{k}\right\}_{k=0,1, \ldots, l-1}$.

Proposition 3 essentially states that if the observations of Problem 1 are generated by a formation $\left\{x_{1}, \ldots, x_{n}\right\}$, then $\mathcal{R}_{i}$ can be assigned to position $p_{i}$ as well as to all the other positions of the subset of $\mathcal{E}_{P_{1}}$ which contains $p_{i}$. This leads to the following results.
Proposition 4 (Number of Solutions). The number of solutions to Problem 1 is

$$
\begin{array}{ll}
(l-1) ! \cdot(l !)^{\frac{n}{l}-1} & \text { if } c \notin P_{1} \\
(l !)^{\frac{n-1}{l}} & \text { if } c \in P_{1} .
\end{array}
$$

Proof: Remember that in all solutions $\mathcal{R}_{1}$ is at $\left(\begin{array}{ll}0 & 0\end{array}\right)^{T}$. If $c \notin P_{1}, \mathcal{E}_{P_{1}}$ has $n / l$ sets, each consisting of $l$ positions. Each set of $\mathcal{E}_{P_{1}}$ has $l$ robots associated, and, in each solution, each of these robots (except for $\mathcal{R}_{1}$ ) can be placed in any position of the set, provided that this position is not occupied by another robot. Hence, $(l-1)$ ! possible permutations correspond to the set of $\mathcal{E}_{P_{1}}$ associated to $\mathcal{R}_{1}$, and $l$ ! possible permutations correspond to the remaining $n / l-1$ sets of $\mathcal{E}_{P_{1}}$. Multiplying these possibilities we obtain (5). A similar analysis leads to (6) if $c \in P_{1}$, noting that $\mathcal{R}_{i}$ associated to the set $\{c\}$ of $\mathcal{E}_{P_{1}}$ has $l$ possible poses if $i \neq 1$.

Corollary 1. For a given $n$, the maximum number of possible solutions to Problem 1 is $(n-1)$ !. This number is actually reached when $P_{1}$ is a regular $n$-gon if $c \notin P_{1}$, and when $P_{1} \backslash c$ is a regular $(n-1)$-gon if $c \in P_{1}$.

Proof: If $c \in P_{1}$ and $l=n-1$ then $(l !)^{\frac{n-1}{l}}=(n-1)$ ! and $P_{1} \backslash c$ is a regular $(n-1)$-gon. If $l<n-1$, then $l$ is a factor of $n-1$ and $m=(n-1) / l \in \mathbb{N}$. Both the numerator and denominator of

$$
r=\frac{(l !)^{m}}{(n-1) !}=\frac{(l !)^{m-1}}{(n-1)(n-2) \ldots(l+1)}
$$

are products of $l(m-1)$ factor,s and the smallest factor of the denominator is larger than the largest factor of the numerator. Then $r<1$, and we can write $(l !)^{\frac{n-1}{l}}<(n-1)$ !. For $c \notin P_{1}$, a similar reasoning leads to $\left(l-1\right.$ !) $(l !)^{\frac{n}{l}-1}<(n-1)$ ! if $l<n$, while if $l=n$ the number of solutions is $(n-1)$ ! and $P_{1}$ is a regular $n$-gon.

Summarizing, each point of $P_{1}$ can be assigned to one and only one subset of partition $\mathcal{E}_{P_{1}}$. If $c \notin P_{1}$, Lemma 1 implies that each subset of $\mathcal{E}_{P_{1}}$ has $l$ positions and $l$ robots assigned to it. Conversely, each robot can assume $l$ different poses which correspond to all the $l$ positions in its subset, with $l$ different orientations that differ by a multiple of $2 \pi / l$. The robots associated to the set to which $\mathcal{R}_{1}$ is associated have only $l-1$ possible poses instead of $l$. Note that all the robots associated to the same set have the observations equal up to a pure rotation. If $c \in P_{1}$ then $\mathcal{R}_{i}(i \neq 1)$ associated to $\{c\}$ has $l$ different possible poses with the same position.

All the solutions are generated by independently permuting the possible poses of each robot, with the constraint that two robots cannot be at the same position. Hence, the set of solutions of Problem 1 is implicitly represented by (1) the set $P_{1}$ (2) the partition $\mathcal{E}_{P_{1}}$ of $P_{1}$ (3) the association between each robot $\mathcal{R}_{i}, i=1, \ldots, n$, and the corresponding set of $\mathcal{E}_{P_{1}}$. This compact representation of the solutions may be used, e.g., to reduce the complexity of MultiReg [10], an algorithm which solves a general class of mutual localization problems that includes Problem 1 as a special case. 


\section{An Anti-Symmetry CONTROL LAW}

Assume that a group of robots must perform a collaborative task which requires mutual localization, and that only anonymous position measures are available. If the robots are initially arranged in a formation resulting in observations that are rotational symmetric, mutual localization will be computationally heavier and will not provide a single solution. In the stochastic case, as mentioned in the introduction, problems will arise whenever the observations are close to being rotational symmetric. For this reason, we introduce in this section a continuous function that measures the distance of sets of points from rotational symmetry. This will be used to design a control law aimed at keeping the solution to Problem 1 unique. We mention that the symmetry distance function proposed in [15] is not practical for our purposes because its computation cannot be executed in real time.

Given the set of points $P_{1}$ and an angle $\phi \in[0,2 \pi)$, define the symmetry metric function

$$
\gamma_{P}(\phi):=e\left(P_{1}, P_{1}(\phi)\right),
$$

where $P_{1}(\phi)$ is defined in (3) and

$$
e\left(P^{\prime}, P^{\prime \prime}\right):=\sum_{p^{\prime} \in P^{\prime}} \min _{p^{\prime \prime} \in P^{\prime \prime}}\left\|p^{\prime}-p^{\prime \prime}\right\|^{2}
$$

is the closest point metric between $P^{\prime}$ and $P^{\prime \prime}$.

Proposition 5 (Properties of $\gamma_{P}$ ). The following statements are true:

1) $\gamma_{P_{1}}(0)=0$.

2) $\gamma_{P_{1}}$ is zero only at $\{2 k \pi / l, k=0, \ldots, l-1\}$, where $l$ is the integer such that $\mathcal{S}_{P}=C_{l}$.

3) $P_{1}$ is rotational symmetric if and only if $\gamma_{P_{1}}$ is zero for some $\phi$ other than 0 .

4) There exist $\phi_{1}, \phi_{2}$, with $0<\phi_{1}<\phi_{2}<2 \pi$, such that $\gamma_{P_{1}}$ is strictly increasing in $\left[0, \phi_{1}\right)$ and strictly decreasing in $\left(\phi_{1}, 2 \pi\right)$.

Proof: 1) is true by definition. Moreover, $\gamma(\phi)=0$ if and only if for any $p^{\prime} \in P_{1}$ exists $p^{\prime \prime} \in P_{1}(\phi)$ s.t. $p^{\prime}=p^{\prime \prime}$. Hence, $P_{1}=P_{1}(\phi)$, i.e., the rotation $R(\phi)$ belongs to $C_{l}$. This implies 2). Also, 2) implies 3). Finally, consider the function $\hat{\gamma}_{P}(\phi)=\sum_{p \in P_{1}}\|(p-c)-R(\phi)(p-c)\|^{2}$, which is equal to $\sum_{p \in P_{1}}(2(p-c) \sin (\phi / 2))^{2}$, that is monotonically increasing in $[0, \pi]$ and monotonically decreasing in $[\pi, 2 \pi]$. For each $p \in P_{1}$ there is a neighborhood of $\phi=0$ in which $\min _{p^{\prime} \in P_{1}(\phi)}\left\|p-p^{\prime}\right\|^{2}=\|(p-c)-R(\phi)(p-c)\|^{2}$, i.e., in which $\gamma_{P}(\phi)=\hat{\gamma}_{P}(\phi)$. Denote by $\Phi \subset[0,2 \pi)$ the set in which $\gamma_{P}(\phi)=\hat{\gamma}_{P}(\phi)$. Then, 4) is proven by taking $\phi_{1}=$ $\max _{\Phi \cap[0, \pi]} \phi$ and $\phi_{2}=\min _{\Phi \cap[\pi, 2 \pi]} \phi$.

As in the proof, define $\phi_{1}=\max _{\Phi \cap[0, \pi]} \phi$ and $\phi_{2}=$ $\min _{\Phi \cap[\pi, 2 \pi]} \phi$. According to Proposition 5, the minimum value of function $\gamma_{P_{1}}$ in the interval $\left[\phi_{1}, \phi_{2}\right]$ (called internal minimum value in the following) is a continuous measure of the distance of $P_{1}$ from being rotational symmetric. If the minimum is zero, $P_{1}$ is actually symmetric. A control action aimed at keeping Problem 1 uniquely solvable can then be based on the strategy of increasing such minimum value.

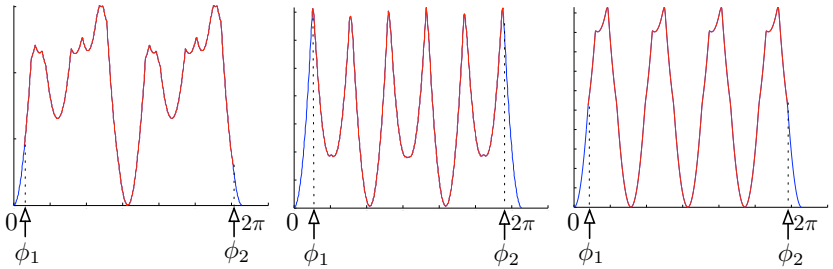

Fig. 4. The symmetry metric function $\gamma$ for the three set of points of Fig. 2, in the same order from left to right.

In particular, assume for simplicity that the position of each robot obeys an omnidirectional kinematic model:

$$
\dot{p}_{i}=u_{i}, \quad i=1, \ldots, n,
$$

where $u_{i}$ is the two-dimensional vector of velocity inputs for $\mathcal{R}_{i}$. Consider the following anti-symmetry control law

$$
u_{i}=\alpha \frac{\bar{p}_{i}-p_{i}}{\left\|\bar{p}_{i}-p_{i}\right\|} \quad i=1, \ldots, n,
$$

where $\alpha$ is a positive gain and

$$
\bar{p}_{i}:=\underset{p \in P_{1}(\bar{\phi})}{\operatorname{argmin}}\left\|p_{i}-p\right\| \quad \bar{\phi}:=\underset{\phi \in\left[\phi_{1}, \phi_{2}\right]}{\operatorname{argmin}} \gamma_{P_{1}}(\phi) .
$$

This control law has a simple interpretation. Once the rotation angle $\bar{\phi}$ that minimizes $\gamma_{P_{1}}$ in $\left[\phi_{1}, \phi_{2}\right]$ has been identified (e.g., numerically), $P_{1}(\bar{\phi})$ is built by rotating $P_{1}$ by $\bar{\phi}$. The closest point $\bar{p}_{i} \in P_{1}(\bar{\phi})$ is found for any $p_{i} \in P_{1}$, and the velocity input is chosen so as push $\mathcal{R}_{i}$ away from $\bar{p}_{i}$ along the segment $p_{i} \bar{p}_{i}$, leading to an increase of $\gamma_{P_{1}}(\bar{\phi})$.

Note that (7) is undefined if $P_{1}$ is rotational symmetric. In this case a simple randomized control can be used for the small time sufficient to break the symmetry.

\section{Simulations}

We have validated the results of Sections III and IV through extensive simulations of the anti-symmetry control law.

The results of the first simulation are shown in Fig. 5 (above). The 9-robot system starts in a lattice formation whose proper symmetry group is $C_{4}$, and moves under the action of the anti-symmetry control. Symmetry is readily broken, as shown by change in symmetry metric function $\gamma_{P_{1}}$, which has 3 internal zeros at start. As the simulation proceeds, the internal minimum value of $\gamma_{P_{1}}$ increases.

Figure 5 (above) also shows the consequence of measurement noise on the accuracy of the estimated solution in the neighborhood of the initial rotational symmetric formation. To compute the solutions of Problem 1, we have used MultiReg, a probabilistic robust estimation algorithm that performs a multiple registration among a set of noisy observations [10]. At each step, we have obtained multiple sets of noisy observations by adding a gaussian noise to the observations of the current arrangement. The figure shows all the possible poses of the circled robot as estimated by MultiReg on the basis of these data. At the start, when the formation is rotational symmetric, the estimated solutions are evenly distributed in 4 clusters of poses. The clusters are centered on all the feasible positions of a single subset of the partition $\mathcal{E}_{P_{1}}$, as predicted by Proposition 3 . The number 

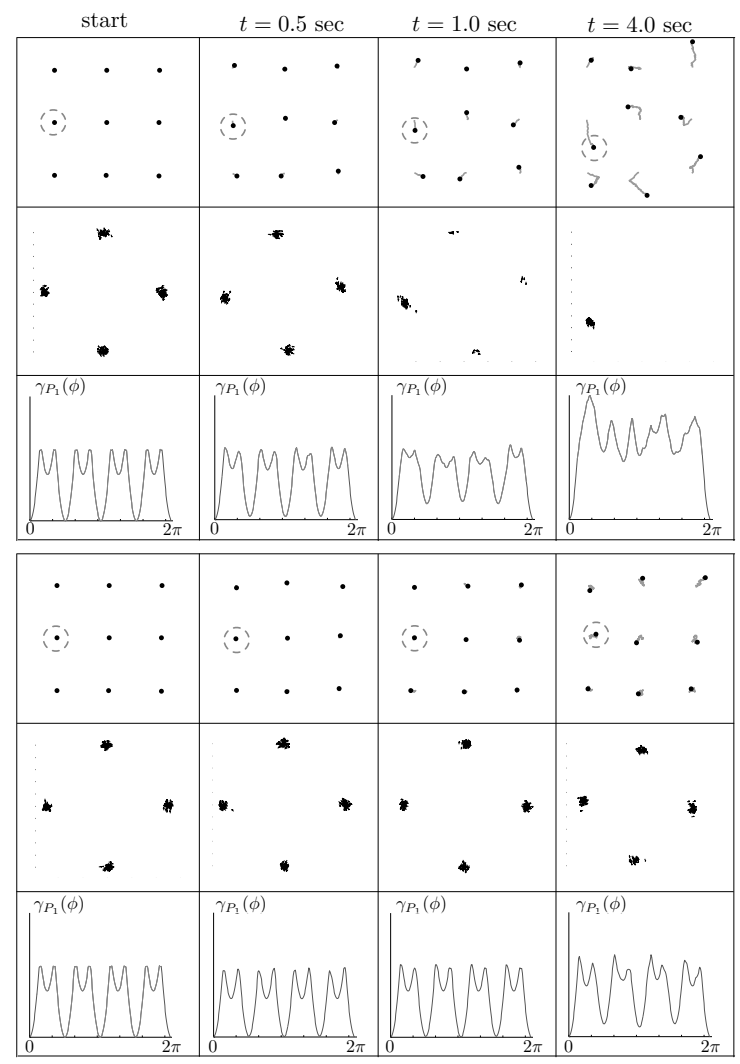

Fig. 5. Above: the use of the anti-symmetry control law to break up a 9-robot lattice formation whose proper symmetry group is $C_{4} ; 4$ snapshots of the formation (top), the estimated positions for the circled robot (center), the symmetry metric function $\gamma_{P_{1}}$ (bottom). Below: the same results with a random control law.

of solutions (576) found by MultiReg matches with the one theoretically derived in Proposition 4. When the symmetry is completely broken, at $t=4.0 \mathrm{~s}$, the surviving estimates have a gaussian distribution centered on the real pose and a covariance comparable to that of the additive noise.

In the intermediate frames, in which the formation is close to being rotational symmetric, the solutions of MultiReg are distributed in more than one cluster, but not evenly. The largest cluster is centered on the real pose of the estimated robot. The other clusters, with less solutions, become feasible configurations only when the additive noise on the observations restores the rotational symmetry.

For comparison, we have also simulated the same 9-robot system under the action of a random control law (Figure 5, below). In fact, since the subset of symmetric configurations has zero measure in the configuration space, a random control law can be expected to break the symmetry. However, the results show that the anti-symmetry control is much more effective in achieving this than the random control. In fact, the increase of the internal minima of $\gamma_{P_{1}}$ with the random control is slower and non-monotonous. Correspondingly, the multiple clusters of the estimation do not disappear.

Other simulations starting from rotational symmetric configurations are shown in Figs. 6-7 and in the accompanying video. See also http://www.dis.uniroma1.it/labrob/ research/mutLoc.html for other material.
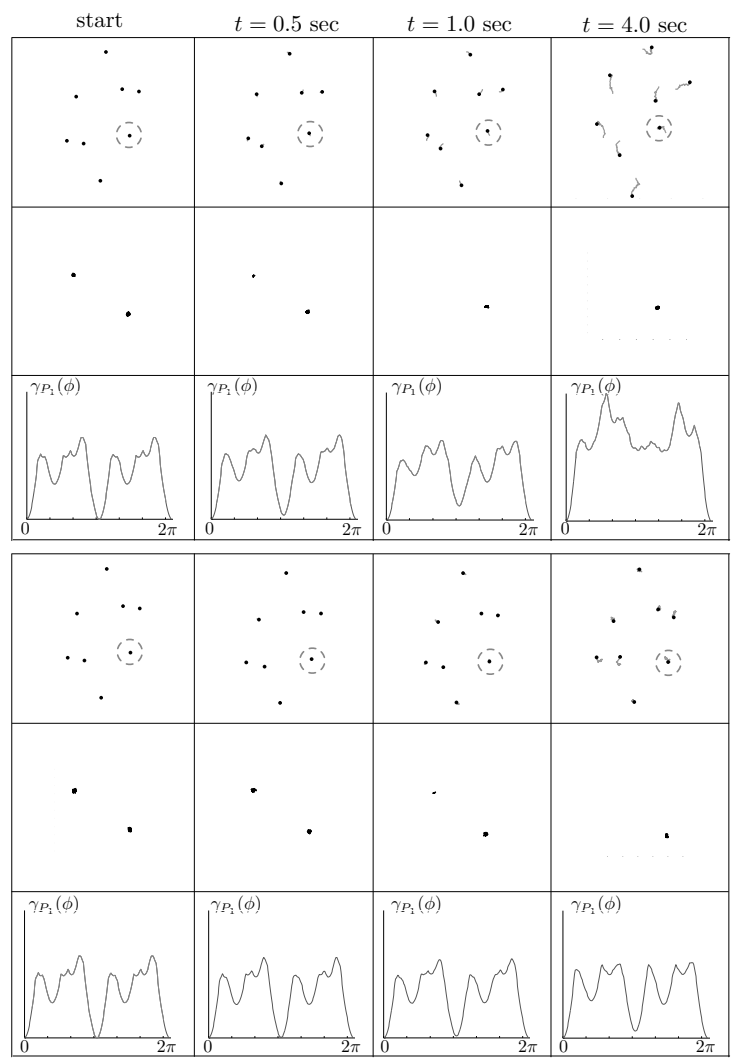

Fig. 6. As in Fig. 5 for a starting formation with proper symmetry group $C_{2}$.
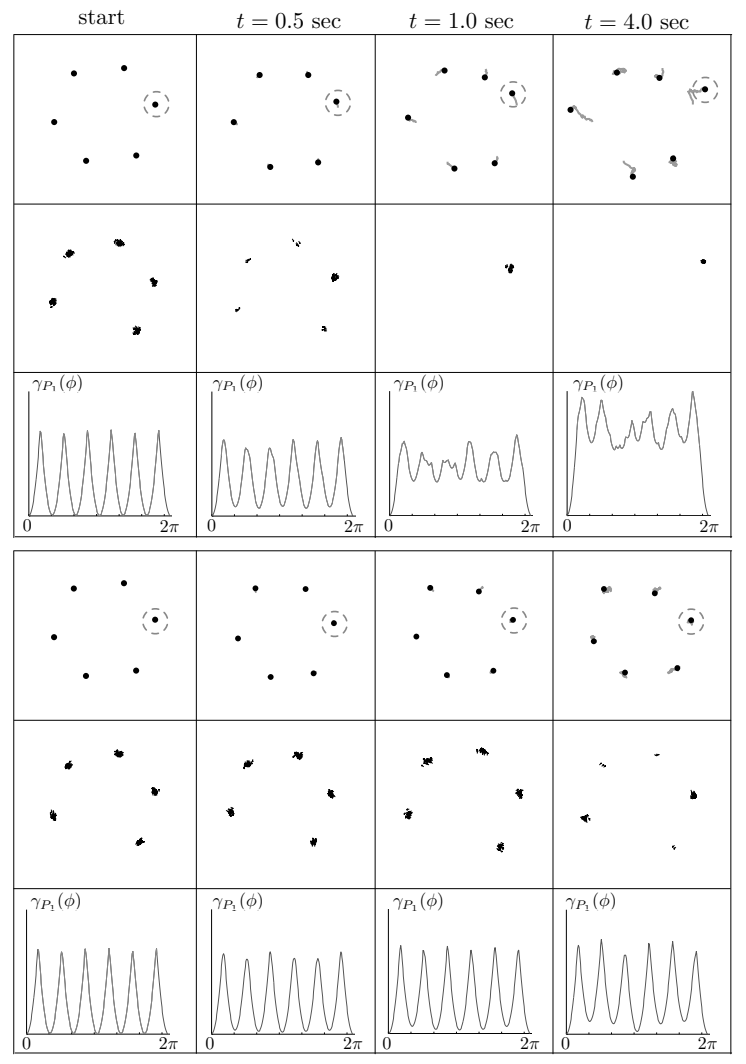

Fig. 7. As in Fig. 5 for a starting formation with proper symmetry group $C_{6}$. 


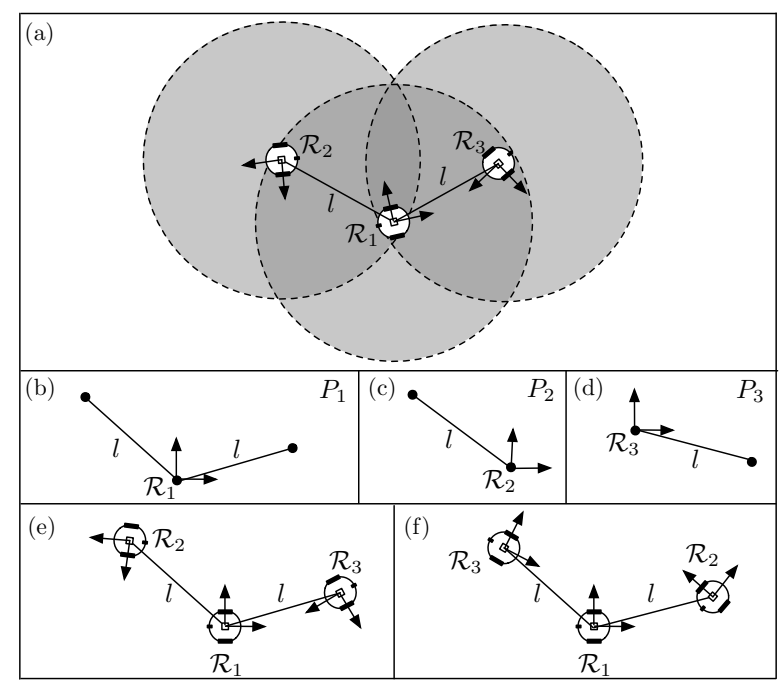

Fig. 8. (a) An example of non-complete measuring graph due to sensor range limitations (b-d) observations of $\mathcal{R}_{1}, \mathcal{R}_{2}$ and $\mathcal{R}_{3}$ (e-f) the two possible solutions, none of them rotational symmetric.

\section{NON-COMPLETE MEASURING GRAPH}

We now sketch an extension of our problem to the case of non-complete measuring graph, i.e., observations that include only subsets of the group of the robots. This situation arises when limited-range or anisotropic sensors are used, or when line-of-sight occlusions occur due to obstacles or robots [10].

Problem 2 (Mutual Localization with Anonymous Position Measures - Generic measuring graph). Given $n$ observations $P_{1}, \ldots, P_{n}$, with $\left|P_{i}\right|=m_{i} \leq n$, find all the sets $\hat{P} \supset P_{1}$, with $|\hat{P}|=n$, together with the associated pairs of functions $\hat{p}:\{2, \ldots, n\} \rightarrow \hat{P} \backslash(00)^{T}, \hat{\theta}:\{2, \ldots, n\} \rightarrow[0,2 \pi)$, with $\hat{p}$ bijective, such that

$$
\hat{P} \ominus \hat{x}_{i} \supset P_{i} \quad i=2, \ldots, n,
$$

where $\hat{x}_{i}:=(\hat{p}(i), \hat{\theta}(i))$.

Proposition 6 (Necessary Condition for Unique Solvability). If Problem 2 admits a solution in which $\hat{P}$ is rotational symmetric, this solution is not unique.

This result is obvious since a solution for which $\hat{P}$ is rotational symmetric generates (by permutation of the robots in the same subset of $\mathcal{E}_{\hat{P}}$ ) additional feasible solutions of Problem 2 that differ for the associated functions $\hat{p}$ and $\hat{\theta}$.

The condition of Proposition 6 is not sufficient. As shown by Fig. 8, there are cases in which multiple solutions exist, but none of them is associated to a rotational symmetric $\hat{P}$.

\section{CONCLUSION AND Future WORKS}

We have theoretically formulated and investigated a novel problem called Mutual Localization with Anonymous Position Measures. This is an extension of Mutual Localization with Position Measures, with the additional assumption that the identities of the measured robots are not known. Through the use of the concept of planar rotational symmetry, we have established a necessary and sufficient condition for the uniqueness of the solution, providing also an associated test. Furthermore, we have studied the structure of multiple solutions, classifying the solutions in a number of equivalence classes which is linear with $n$, and determined as $(n-1)$ ! the upper bound to the maximum number of possible solutions.

Through the introduction of a continuous function that measures the distance of sets of points from rotational symmetry, we have designed a control law aimed at keeping the solution to Problem 1 unique. Its superior performance in comparison with a random control was confirmed by extensive simulation. The application of a multiple registration algorithm (Multireg) corroborates the theoretical results about number and structure of the solutions and suggests some interesting probabilistic considerations.

Current work is aimed at a comprehensive treatment of the case of generic measuring graph as well as at the design of a decentralized anti-symmetry control.

\section{REFERENCES}

[1] J. Aspnes, T. Eren, D. K. Goldenberg, A. S. Morse, W. Whiteley, Y. R. Yang, B. D. O. Anderson, and P. N. Belhumeur, "A theory of network localization," IEEE Transactions on Mobile Computing, vol. 5, no. 12, pp. 1663-1678, 2006.

[2] T. Eren, W. Whiteley, and P. Belhumeur, "Using angle of arrival (bearing) information in network localization," in 45th IEEE Conf. on Decision and Control, San Diego, CA, Jan. 2006, pp. 4676-4681.

[3] G. Piovan, I. Shames, B. Fidan, F. Bullo, and B. Anderson, "On frame and orientation localization for relative sensing networks," in 47th IEEE Conf. on Decision and Control, Cancun, Mexico, Dec. 2008, pp. 2326-2331.

[4] M. Batalin and G. Sukhatme, "The design and analysis of an efficient local algorithm for coverage and exploration based on sensor network deployment," IEEE Transactions on Robotics, vol. 23, no. 4, pp. 661675, Aug 2007.

[5] A. Franchi, L. Freda, G. Oriolo, and M. Vendittelli, "The sensor-based random graph method for cooperative robot exploration," IEEE/ASME Transactions on Mechatronics, vol. 14, no. 2, pp. 163-175, 2009.

[6] R. Sepulchre, D. A. Paley, and N. E. Leonard, "Stabilization of planar collective motion: All-to-all communication," IEEE Transactions on Automatic Control, vol. 52, no. 5, pp. 811-824, May 2007.

[7] D. B. Kingston, R. W. Beard, and R. S. Holt, "Decentralized perimeter surveillance using a team of UAVs," IEEE Transactions on Robotics, vol. 24, no. 6, pp. 1394-1404, 2008.

[8] G. Antonelli, F. Arrichiello, and S. Chiaverini, "The entrapment/escorting mission: An experimental study using a multirobot system," IEEE Robotics \& Automation Magazine, vol. 15, no. 1, pp. 22-29, 2008.

[9] X. Zhou and S. Roumeliotis, "Robot-to-robot relative pose estimation from range measurements," IEEE Transactions on Robotics, vol. 24, no. 6, pp. 1379-1393, 2008.

[10] A. Franchi, G. Oriolo, and P. Stegagno, "Mutual localization in a multirobot system with anonymous relative position measures," in 2009 IEEE/RSJ Int. Conf. on Intelligent Robots and Systems, St. Louis, MO, Oct. 2009, pp. 3974-3980.

[11] F. Lu and E. Milios, "Globally consistent range scan alignment for environment mapping," Autonomous Robots, vol. 4, no. 4, pp. 333349, 1997.

[12] M. Montemerlo and S. Thrun, "Simultaneous localization and mapping with unknown data association using fastslam," in 2003 IEEE Int. Conf. on Robotics and Automation, Taipei, Taiwan, Sep. 2003, pp. 1985-1991.

[13] R. C. Smith and P. Cheeseman, "On the representation and estimation of spatial uncertainly," International Journal of Robotics Research, vol. 5, no. 4, pp. 56-68, 1986.

[14] W. Miller, Symmetry Groups and Their Applications. Academic Press, 1972.

[15] H. Zabrodsky, S. Peleg, and D. Avnir, "Symmetry as a continuous feature," IEEE Transactions on Pattern Analysis \& Machine Intelligence, vol. 17, no. 12, pp. 1154-1166, 1995. 